\title{
Vorbereitende Bedingungen von Sprechakten und mentale Räume
}

\author{
Enrique Huelva Unternbäumen (Brasilia)
}

\begin{abstract}
In this paper I shall analyze interaction sequences in which the interactants explicitly thematize and problematize whether the preparatory conditions of a speech act in a specific enunciation situation can be considered as satisfied or not. In these sequences the interaction develops an open, and consequently, observable contextualization process. In order to describe the dynamics of this process, central concepts and methods of Mental Space Theory will be applied. Contextualization will be characterized as a continuous process of construction, connection and modification of mental spaces.
\end{abstract}

\section{$1 \quad$ Einleitung}

Vorbereitende Bedingungen von Sprechakten können prinzipiell auf zwei unterschiedlichen Ebenen untersucht und charakterisiert werden. Auf der Ebene von Sprechakttypen stellen sie sprechakttypische Einschränkungen des Kontextes dar. Ein Versprechen macht beispielsweise nur dann Sinn, wenn die versprochene Handlung seitens des Hörers auch erwünscht ist, oder eine Aufforderung zu einer Tätigkeit, wenn diese nicht ohnehin schon vollzogen wird. Die Ermittlung und Charakterisierung von solchen allgemeinen kontextuellen Einschränkungen für Sprechaktfamilien bzw. für einzelne Sprechakttypen ist eine der zentralen Aufgaben der Illokutionslogik (cf. etwa Vanderveken 1988: 165 ff.; Rolf 1997: 134 ff.). Auf der Ebene von einzelnen Sprechakten nehmen vorbereitende Bedingungen die Gestalt von (tatsächlich realisierten) Sprecher-Präsuppositionen an. Vorbereitende Bedingungen sind auf dieser Ebene vom Sprecher als gegeben unterstellte, präsupponierte Zustände eines jeweils konkreten Äußerungskontextes. Im Vollzug eines bestimmten Sprechakts präsupponiert der Sprecher, dass gewisse Zustände, die für die defektfreie Ausführung des Sprechakts notwendig sind, im konkreten Kontext des Äußerungsakts gegeben sind. Als Aspekte einer bestimmten, tatsächlichen Äußerungssituation sind vorbereitende Bedingungen in diesem zweiten Sinne ein für die (sprechakttheoretisch fundierte) Dialoganalyse ${ }^{1}$ relevanter Untersuchungsgegenstand.

Betrachten wir zur Verdeutlichung dieser Unterscheidung das folgende Beispiel:

\footnotetext{
${ }^{1}$ Einen Überblick über dieses theoretisch und methodologisch relativ heterogene Forschungsgebiet finden wir in Fritz/Hundsnurscher (eds.) (1994).
} 
$(\mathbf{1})^{2}$

$\begin{array}{rrr}12 & C: & \text { creo que uno lo trajo enrique para él } \\ 13 & \text { C: } & \text { porque yo había dicho que tomaba del tuyo } \\ 14 & \text { B: } & \text { ah: : verdad }\end{array}$

Durch die Äußerung der Zeile 12 im Beispiel (1) führt die Sprecherin C einen Sprechakt der Familie der Assertiva aus. Es handelt sich dabei konkret um einen Sprechakt des Typs eine Vermutung äußern (cf. Vanderveken 1988, 169; 1990: 172; Rolf 1997, 143-144). Als assertiver Sprechakt zeichnet sich der Sprechakttyp eine Vermutung äußern durch die vorbereitende Bedingung $\Sigma_{l}$ aus, die besagt, dass der Sprecher Evidenzen (Gründe, Belege, o. Ä.) dafür hat, dass die von ihm ausgedrückte Proposition $\mathrm{P}$ einen wahrscheinlich bestehenden Sachverhalt repräsentiert (cf. Rolf 1997, 144). Im konkreten Äußerungskontext i von (1) determiniert $\Sigma_{l}$, dass die Sprecherin $\mathrm{C}$ die Proposition $\mathrm{P}_{1}=\{$ die Sprecherin $\mathrm{C}$ hat Evidenzen (Gründe, Belege) dafür, dass die Proposition ihrer Äußerung $\mathrm{P}=\{$ enrique trajo un café para él\} einen wahrscheinlich bestehenden Sachverhalt repräsentiert\} als wahr präsupponieren muss. Durch den Vollzug des Sprechakts der Zeile 12 beansprucht die Sprecherin C implizit und automatisch die Geltung ${ }^{3}$ des durch die präsupponierte Proposition $\mathrm{P}_{1}$ ausgedrückten Sachverhalts als konstitutiven Bestandteil des Äußerungskontexts. Oder anders ausgedrückt: die Sprecherin C führt in 12 nicht nur einen Sprechakt des Typs eine Vermutung äußern aus, sondern trägt zugleich zur Konstruktion des Kontextes bei, in den sich dieser Sprechakt einbettet, indem sie den Inhalt von $\mathrm{P}_{1}$ als einen Aspekt dieses Kontextes präsentiert. $\mathrm{C}$ führt also eine sprachliche Handlung aus und, indem sie das tut, kontextualisiert sie sie zugleich.

In den meisten Fällen ist das Erfülltsein von vorbereitenden Bedingungen bzw. die (intersubjektive) Gültigkeit des durch sie präsupponierten Sachverhalts nicht strittig. Der Hörer ratifiziert sie stillschweigend: Indem die Nachfolgeäußerung, mit der der Hörer auf die sprachliche Handlung des Sprechers reagiert, die vorbereitenden Bedingungen dieser sprachlichen Handlung weder direkt noch indirekt thematisiert, bestätigt er, dass auch für ihn die vorbereitenden Bedingungen dieser Handlung als erfüllt gelten. Im Beispiel (1) signalisiert der Sprecher B durch seine Äußerung in der Zeile 14 Verstehen bzw. Verständigung und ratifiziert implizit, dass der durch $\mathrm{P}_{1}$ ausgedrückte Sachverhalt $=\{$ die Sprecherin C hat Evidenzen (Gründe, Belege) dafür, dass die Proposition ihrer Äußerung P =

\footnotetext{
2 Das Beispiel stammt aus Huelva Unternbäumen (2005). Die deutsche Übersetzung könnte lauten:

12: C: ich glaube, einen hat enrique für sich selber mitgebracht

13: C: weil, ich hatte ihm gesagt, dass ich aus deinem trinken würde

14: B: achso

3 In Anlehnung an Schnädelbach (1977: 177ff.) soll hier und im Folgenden unter 'Geltung' die Dimension zu verstehen sein, in der bestimmte Ansprüche und Bedingungen charakterisiert werden, ohne dass sie gerechtfertigt, eingelöst oder erfüllt wären. 'Gültigkeit' soll dagegen denjenigen (Dialog-)Zustand bezeichnen, in dem Legitimität von Geltungsansprüchen und das Erfülltsein von Bedingungen bereits bestehen.
} 
\{enrique trajo un café para él\} einen wahrscheinlich bestehenden Sachverhalt repräsentiert\} auch für ihn einen gültigen Bestandteil des Äußerungskontextes von 12 darstellt. ${ }^{4}$

Das ist jedoch nicht immer der Fall. Es gibt durchaus Fälle, in denen der Sprecher und/oder der Hörer explizit thematisieren, ob die vorbereitenden Bedingungen eines bereits vollzogenen bzw. eines noch zu vollziehenden Sprechakts (cf. dazu Huelva Unternbäumen 2005) als erfüllt (bzw. als intersubjektiv gültig) gelten (sollen) oder nicht. In solchen Fällen entwickelt die Interaktion einen offenen und folglich auch beobachtbaren Prozess der Hervorbringung von Kontext, einen offenen, beobachtbaren Kontextualisierungsprozess. Sprecher und Hörer handeln metakommunikativ in dem Sinne, dass sie die intersubjektive Gültigkeit, die gegenseitige Anerkennung des Erfülltseins von vorbereitenden Bedingungen bzw. des durch sie ausgedrückten Sachverhalts zum Gegenstand ihrer jeweiligen Äußerungen machen. Die zentrale kommunikative Absicht ihrer Äußerungen ist dann die Stiftung von Konsens hinsichtlich der Gültigkeit einer bestimmten vorbereitenden Bedingung oder einfach die Stiftung von Konsens darüber, dass ein solcher Konsens halt nicht besteht. Gegenstand des vorliegenden Beitrags ist die Beschreibung der Dynamik des Prozesses der Konstruktion von Kontext, die in solchen Interaktionsstellen entsteht.

Ein solches Unterfangen bereitet gewisse theoretisch-methodologische Probleme. Wenn wir Kontext im Allgemeinen und die in Form von vorbereitenden Bedingungen thematisierten Kontextaspekte im Besonderen nicht als material gegebene, außersprachliche Größen betrachten, sondern von einem kognitiven Kontextbegriff ausgehen (cf. dazu u. a. Auer (1986; 1992) und Gumperz 1982; 1990; 1992) und folglich Kontext und die in Form von vorbereitenden Bedingungen thematisierten Kontextaspekte als interaktiv geleitete, kognitive Konstrukte der Interaktionsteilnehmer konzeptualisieren und beschreiben wollen, dann brauchen wir einen theoretisch-methodologischen Rahmen, der mindestens den beiden folgenden Anforderungen gerecht wird:

1. Er muss es uns ermöglichen, den Inhalt und die innere Struktur dieser mentalen Konstrukte zu beschreiben.

2. Er muss es uns ermöglichen, die Dynamik der Hervorbringung und Modifikation dieser Konstrukte im Laufe des Interaktionsprozesses zu beschreiben.

In dem vorliegenden Beitrag soll gezeigt werden, dass uns die Theorie der mentalen Räume (Fauconnier 1994; 1997) ein theoretisch-methodologisches Instrumentarium zur Verfügung stellt, das diesen beiden Anforderungen gerecht wird. Es soll also gezeigt werden, dass sie beobachtungsadäquat ist in dem Sinne, dass sie die kognitive Dimension und die beobachtbare Dynamik der Hervorbringung und kontinuierlichen Modifikation der hier relevanten Kontextaspekte konzeptuell voll erfasst. Mit der Anwendung dieser Theorie auf unseren Untersuchungsgegenstand gehen einige zentrale konzeptuelle Implikationen einher, die ich hier kurz ansprechen möchte (cf. auch unten Abschnitt 3).

\footnotetext{
${ }^{4}$ Das ist sicherlich der einfachste Fall der Kontextkoordination, um ein Begriff der Theorie der Verständigungsprobleme zu verwenden (cf. Kindt 2002: 28ff). Ich abstrahiere hier zunächst von der sprechaktbezogenen Funktion des Kausalsatzes der Zeile 13. Cf. dazu Huelva Unternbäumen (2005).
} 
Der konkrete Kontext, in den ein bestimmter Sprechakt eingebettet wird und der für dessen defektfreien Vollzug notwendig ist, wird als mentaler Raum konzeptualisiert. Mentale Räume werden als "the domains that discourse builds up to provide a cognitive substrate for reasoning and for interfacing with the world" definiert (Fauconnier 1997: 34). Intern sind mentale Räume durch frames, kognitive Modelle und aus ihnen resultierendes Inferenzwissen strukturiert (cf. Fauconnier 1997: 39 ff; zum Konzept von frame cf. Fillmore 1982, 1985; zum Konzept von kognitivem Modell cf. Lakoff 1987). Wir gehen also im Folgenden davon aus, dass im Vollzug eines bestimmten Sprechakts der Sprecher einen mentalen Raum aufbaut, der in Gestalt von frames, kognitiven Modellen und Inferenzen die mentale Repräsentation jener konkreten Zustände und Sachverhalte umfasst, die für den defektfreien Vollzug des Akts notwendig und ausreichend sind. Dazu gehören unter anderem jene vom Sprecher als gegeben präsupponierte Zustände und Sachverhalte, die dazu dienen, das Erfülltsein der vorbereitenden Bedingungen des vollzogenen Akts zu beanspruchen.

Die Nachfolgeäußerungen, mit denen der Hörer auf den vollzogenen Sprechakt reagiert, können Struktur und Inhalt des im Vollzug dieses Sprechakts aufgebauten mentalen Raums verändern. Eine Nachfolgeäußerung kann die Konstruktion eines neuen mentalen Raums bewirken, der mit dem Ursprungsraum (Basisraum) mit Hilfe von (mentalen) Konnektoren verbunden ist und der einen Inhalt und eine Struktur aufweist, die sich von dem Inhalt und der Struktur des Basisraums unterscheiden (cf. Fauconnier 1997: 37 ff). Ein Gespräch kann aus dieser Sicht als "a succession of cognitive configurations" (Fauconnier 1997: 38) betrachtet werden. Die uns hier interessierenden Fälle zeichnen sich dadurch aus, dass jene Elemente des Basisraums durch einen Nachfolgeraum modifiziert werden, die die vom Sprecher als gegeben präsupponierten Zustände und Sachverhalte repräsentieren, jene Einheiten also, auf die er sich stützt, um (unausgesprochen) das Erfülltsein der vorbereitenden Bedingungen des vollzogenen Akts zu beanspruchen.

Im Folgenden soll anhand der Analyse eines konkreten Beispiels die angesprochene Dynamik der Konstruktion und Modifikation von mentalen Räumen, die durch die Wirkung des Sprechaktvollzugs und der Nachfolgeäußerungen entsteht und die Konsequenzen, die sich aus dieser Dynamik für das Erfülltseins der vorbereitenden Bedingungen des vollzogenen Sprechakts ergeben, genauer dargelegt werden.

\section{Die Dynamik der interaktiven Konstruktion mentaler Räume}

Das folgende Beispiel stammt aus Fauconnier (1997: 120ff und 164). Die dort (mit einer anderen Zielsetzung) durchgeführte Analyse wird im Folgenden in leicht vereinfachter Form wiedergegeben. Die für uns relevante Frage wird sein, wie sich die konstatierte Dynamik der Konstruktion von mentalen Räumen auf den ersten Sprechakt der Interaktionssequenz auswirkt. Bevor wir auf die Analyse von Fauconnier eingehen, soll im Folgenden die illokutionslogische Struktur dieses Sprechakts - jedenfalls hinsichtlich der für uns relevanten Aspekte - kurz beschrieben werden. 
$(2)^{5}$

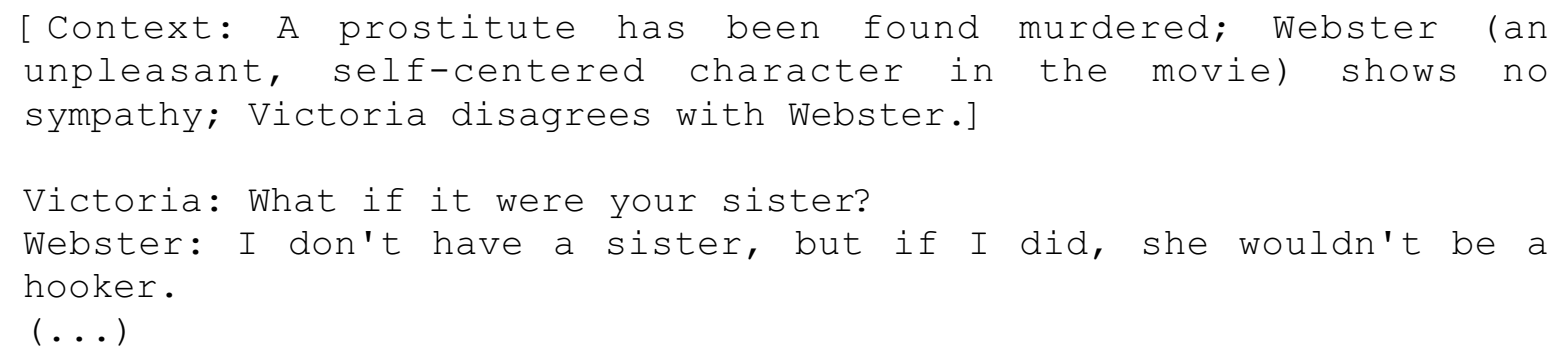

Durch ihre erste Äußerung im Beispiel (2) führt Victoria einen Sprechakt der Familie der Expressiva aus. Es handelt sich dabei konkret um einen Sprechakt des Typs jmdm. einen Vorwurf machen (cf. u.a. Hundsnurscher 1993; Rolf 1997: 238-239). Als expressiver Sprechakt zeichnet sich der Sprechakttyp jmdm. einen Vorwurf machen durch die familienallgemeine vorbereitende Bedingung $\Sigma_{l}$ aus, die besagt, dass ein bestimmtes Ereignis stattgefunden hat, ein Ereignis, von dem entweder der Sprecher oder der Hörer betroffen bzw. für dessen Zustandekommen entweder der einer oder der andere verantwortlich ist (cf. Rolf 1997: 216). ${ }^{6}$ Ferner hat dieser Sprechakttyp als spezifische vorbereitende Bedingungen $\Sigma_{2}$, wonach der Sprecher von einem bestimmten Sachverhalt betroffen ist, und $\Sigma_{3}$, wonach der Hörer in den Augen des Sprechers für das Zustandekommen dieses Sachverhalts verantwortlich ist (cf. Rolf 1997: 238-239). Hundsnurscher (1993) und Rolf (1997: 238f.) weisen außerdem darauf hin, dass der Sprechakttyp jmdm. einen Vorwurf machen gewisse Ähnlichkeiten mit Assertiva und mit Direktiva aufweist. Denn auf der einen Seite ist ein Vorwurf "eine Art Aufforderung, und zwar eine Aufforderung, sich zu entschuldigen oder sich zu rechtfertigen" (Hundsnurscher 1993: 143) bzw. eine Aufforderung, "nicht zu wiederholen, was Anlass zu dem Vorwurf gegeben hat" (Rolf 1997: 239). Und auf der anderen Seite "ist, wer einen Vorwurf erhebt, auf die Behauptung der von ihm thematisierten Proposition illokutionär festgelegt" (Rolf 1997: 239).

Im konkreten Äußerungskontext $\mathrm{i}$ von (2) determiniert $\Sigma_{1}$, dass Victoria die Proposition $\mathrm{P}_{1}=$ $\{$ Victoria hat Gründe, Evidenzen, Belege o. Ä. dafür, dass die von ihr thematisierte Proposition \{what if it were your sister?\} sich auf ein bestimmtes Ereignis bezieht, das (tatsächlich) stattgefunden hat und von dem entweder sie oder Webster betroffen bzw. für dessen Zustandekommen entweder der eine oder der andere verantwortlich ist\} als wahr präsupponieren muss. Das präsupponierte Ereignis besteht darin, dass Webster eine bestimmte, von Victoria als erwartbar betrachtete Handlung nicht vollzogen hat: Er hat nämlich beim Anblick der ermordeten Prostituierte kein Mitgefühl, Mitleid, etc. gezeigt (zu

\footnotetext{
5 Es handelt sich um ein Gespräch aus dem Film The Naked Lie. Die deutsche Übersetzung könnte lauten: (Kontext: Eine Prostituierte wurde ermordert gefunden. Webster (ein unfreundicher und egozentrischer Charakter in dem Film) zeigt kein Mitleid. Victoria zeigt sich verägert über dieses Verhalten.) 
der Auffassung, dass auch Handlungen als Ereignisse aufgefasst werden können, cf. Davidson 1985: 74ff.). Durch den Vollzug des Vorwurfs präsupponiert Victoria nach $\Sigma_{2}$ außerdem, dass sie vom Ausbleiben der erwartbaren Handlung betroffen ist und nach $\Sigma_{3}$, dass Webster in ihren Augen für die unterlassene Handlung verantwortlich ist. ${ }^{7}$

Nach Fauconnier (1997: 121) dienen die zwei ersten Gesprächsbeiträge in (2) "to negotiate space building". Er charakterisiert sie "as a competition between the participants to build appropriate spaces and connections, and thereby obtain intended inferences within the discourse" (Fauconnier 1997: 123). Im Folgenden soll gezeigt werden, dass es den Gesprächsteilnehmern bei dieser "competition" vor allem um den Zustand der vorbereitenden Bedingungen des im ersten Redebeitrag erhobenen Vorwurfs geht. Victoria baut im Vollzug ihrer Vorwurfshandlung im ersten Redebeitrag einen mentalen Raum auf, in dem $\Sigma_{1}, \Sigma_{2}$ und $\Sigma_{3}$ als erfüllt dargestellt werden. Webster seinerseits konstruiert in seinem Nachfolgebeitrag einen neuen mentalen Raum, der den Inhalt und die Struktur des ersten Raums derart modifiziert, dass $\Sigma_{1}, \Sigma_{2}$ und $\Sigma_{3}$ als unerfüllt präsentiert werden. Betrachten wir diesen Konstruktionsprozess Schritt für Schritt.

Ausgehend von einem Basisraum (B) (Realitätsraum) bildet Victoria durch ihren ersten Redebeitrag (vor allem durch die Wirkung der Konjunktion if) einen kontrafaktischen mentalen Raum (M), dessen Elemente mit Elementen des Basisraums verbunden sind (cf. Figur 1):

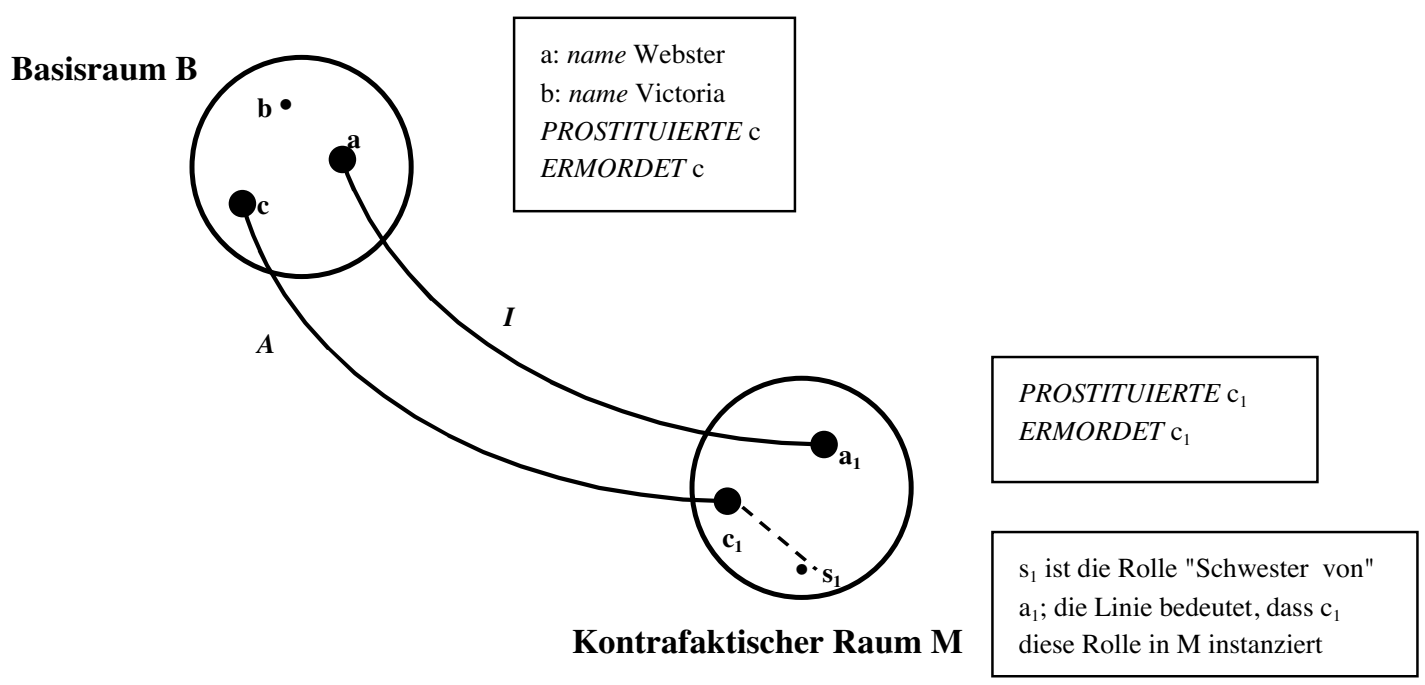

Figur 1

Wie in Figur 1 dargestellt, enthält der Basisraum drei Elemente. Die Elemente a und b sind jeweils mit den Namen Webster und Victoria verbunden. Das Element c wird seinerseits mit

\footnotetext{
7 Diese vorbereitende Bedingung legt fest, dass für das Zustandekommen des thematisierten Sachverhalts ausschliesslich der Unwille - und nicht etwa das Unvermögen - des Adressaten verantwortlich ist: Webster zeigt kein Mitgefühl, weil er es nicht will und nicht etwa, weil er es nicht kann o. Ä.
} 
zwei unterschiedlichen frames assoziiert, nämlich mit den frames PROSTITUIERTE und ERMORDET. Das Element c instanziert jeweils einen bestimmten Bestandteil dieser frames. Der kontrafaktischer Raum bildet die Gegenstücke $\mathrm{a}_{1}$ und $\mathrm{c}_{1}$ zu den Elementen a und c des Basisraums und importiert von diesem die frames PROSTITUIERTE und ERMORDET. Intern ist der kontrafaktische Raum durch diese frames strukturiert. Außerdem enthält der kontrafaktische Raum ein neues Element: $s_{1}$. $S_{1}$ steht für die Rolle "Schwester von $a_{1}$ ". Diese Rolle wird durch das Element $c_{1}$ ausgeführt. Die Elemente a und c des Basisraums und ihre Gegenstücke in dem kontrafaktischen Raum werden jeweils durch unterschiedliche Konnektoren in Verbindung gebracht. Die Verbindung zwischen a und $\mathrm{a}_{1}$ wird durch den Identitätskonnektor $\boldsymbol{I}$ hergestellt, der besagt, dass $\mathrm{a}_{1}$ mit derselben Einheit verbunden ist als a im Basisraum (also mit dem Name Webster). Die Elemente $\mathrm{c}$ und $\mathrm{c}_{1}$ sind dagegen nicht mithilfe des Identitäts-, sondern des Analogiekonnektors $\boldsymbol{A}$ miteinander verbunden. Die Wirkung dieses Konnektors legt eine Interpretation der ersten Äußerung von Victoria nahe, die, so Fauconnier (1997: 121), folgendermaßen paraphrasiert werden kann: "What if, instead of happening to this girl, it had happened to your sister?". Der von Victoria im Vollzug des Sprechakts des ersten Gesprächsbeitrags konstruierte kontrafaktische Raum "ersetzt" sozusagen das wirkliche Mädchen analogisch durch die Schwester von Webster.

Die Konstruktion dieses kontrafaktischen Raums führt nun zur Aktivierung von neuen frames und kognitiven Modellen des Typs 'man muss Mitgefühl zeigen, wenn die eigene Schwester ermordet wird' (cf. Fauconnier 1997: 122f.). Aus diesen allgemeinen frames und kognitiven Modellen werden im kontrafaktischen Raum M Inferenzen für die Interpretation des in ihm dargestellten Sachverhalts abgeleitet. Aufgrund der Verbindung zwischen kontrafaktischem und Basisraum fließen diese Inferenzen zurück zum Basisraum. Figur 2 stellt diesen Sachverhalt grafisch dar.

Basisraum B

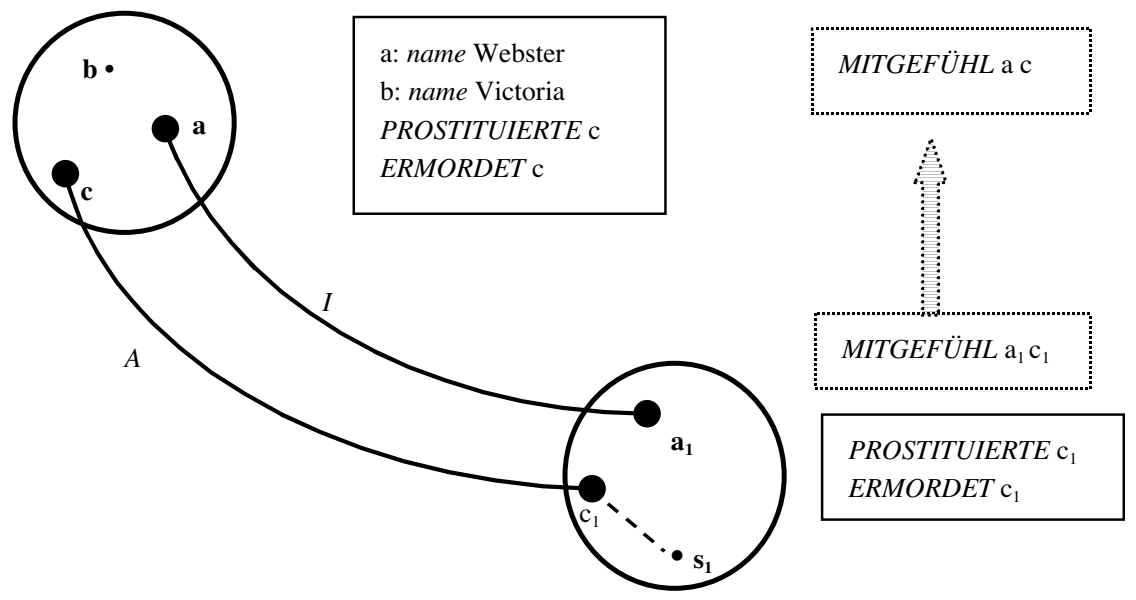

Kontrafaktischer Raum M

Figur 2 
Die Konstruktionsleistung des ersten Redebeitrags von Victoria zeichnet sich durch einen letzten wichtigen Aspekt aus. Victoria behauptet zwar nicht, dass Webster eine Schwester hat, aber die Verwendung des definiten Ausdrucks your sister kann so interpretiert werden, dass sie es präsupponiert (cf. Fauconnier 1997: 122f.). So aufgefasst, bewirkt der Ausdruck your sister die Einführung eines neuen Elements $c^{\prime}$ (= Websters Schwester) in den Basisraum, der mit dem Element $\mathrm{c}_{1}$ des kontrafaktischen Raums mittels des Identitätskonnektors verbunden wird (cf. Figur 3). ${ }^{8}$

Wie Figur 3 zeigt, ist die Rolle "Schwester von Webster" in dem kontrafaktischen und in dem Basisraum jeweils durch die Elemente $\mathrm{s}_{1}$ und s repräsentiert. Diese Elemente sind durch den Identitätskonnektor $\boldsymbol{I}$ miteinander verbunden. Auch ihre jeweilige Werte $\mathrm{c}_{1}$ und $\mathrm{c}^{\prime}$ sind, wie bereits erwähnt, durch den Identitätskonnektor $I$ miteinander verbunden. $\mathrm{C}_{1}$ ist allerdings noch mit einem weiteren Element des Basisraums mittels des analogischen Konnektors $\boldsymbol{A}$ verbunden, nämlich mit c (= die Prostituierte). Wir haben also einen Fall von Doppelverbindung: ein Element eines der Räume wird mit mehr als einem Element des anderen Raums verbunden. Diese Doppelverbindung bewirkt einen so genannten frameblending effect: ${ }^{9} \mathrm{C}_{1}$ ist mit zwei Elementen verbunden, die in zwei unterschiedlichen frames integriert sind, nämlich mit dem Element $\mathrm{c}$, das einen Bestandteil des frames (ERMORDETE) PROSTITUIERTE instanziert und mit $\mathrm{c}^{\prime}$, das in dem frame SCHWESTER VON WEBSTER integriert ist. Konsequenterweise können relevante Eigenschaften von $\mathrm{c}_{1}$ teilweise aus den Eigenschaften dieser beiden frames abgeleitet werden. Durch die Wirkung des Analogiekonnektors $\boldsymbol{A}$ fließen diese Eigenschaften zurück zum Basisraum, so dass "we get the inference intended by Victoria that Webster should show sympathy for the murdered woman" (Fauconnier 1997: 123).

8 Die Präsupposition \{Webster hat eine Schwester\} ist kein Bestandteil des kontrafaktischen Raums, sondern nur des Basisraums (Realitätsraums). Victoria konstruiert einen Realitätsraum, in dem dargestellt wird, dass Webster tatsächlich eine Schwester hat. Zur Beziehung zwischen mentalen Räumen und 'realer' Welt cf. Fauconnier (1997: 34-37). Zum Problem der Projektion von Präsuppositionen cf. Fauconnier (1997: 60-64).

9 "Blending" wird definiert als eine kognitive Operation, die eine teilweise Integration von Strukturen aus zwei unterschiedlichen kognitiven Bereichen bewirkt, so dass ein dritter kognitiver Bereich mit eigenen, emergenten Eigenschaften entsteht. Cf. dazu Fauconnier (1997: 149-186); Fauconnier/Turner (1996); Fauconnier/Turner (1998); Fauconnier/Turner (2002). 

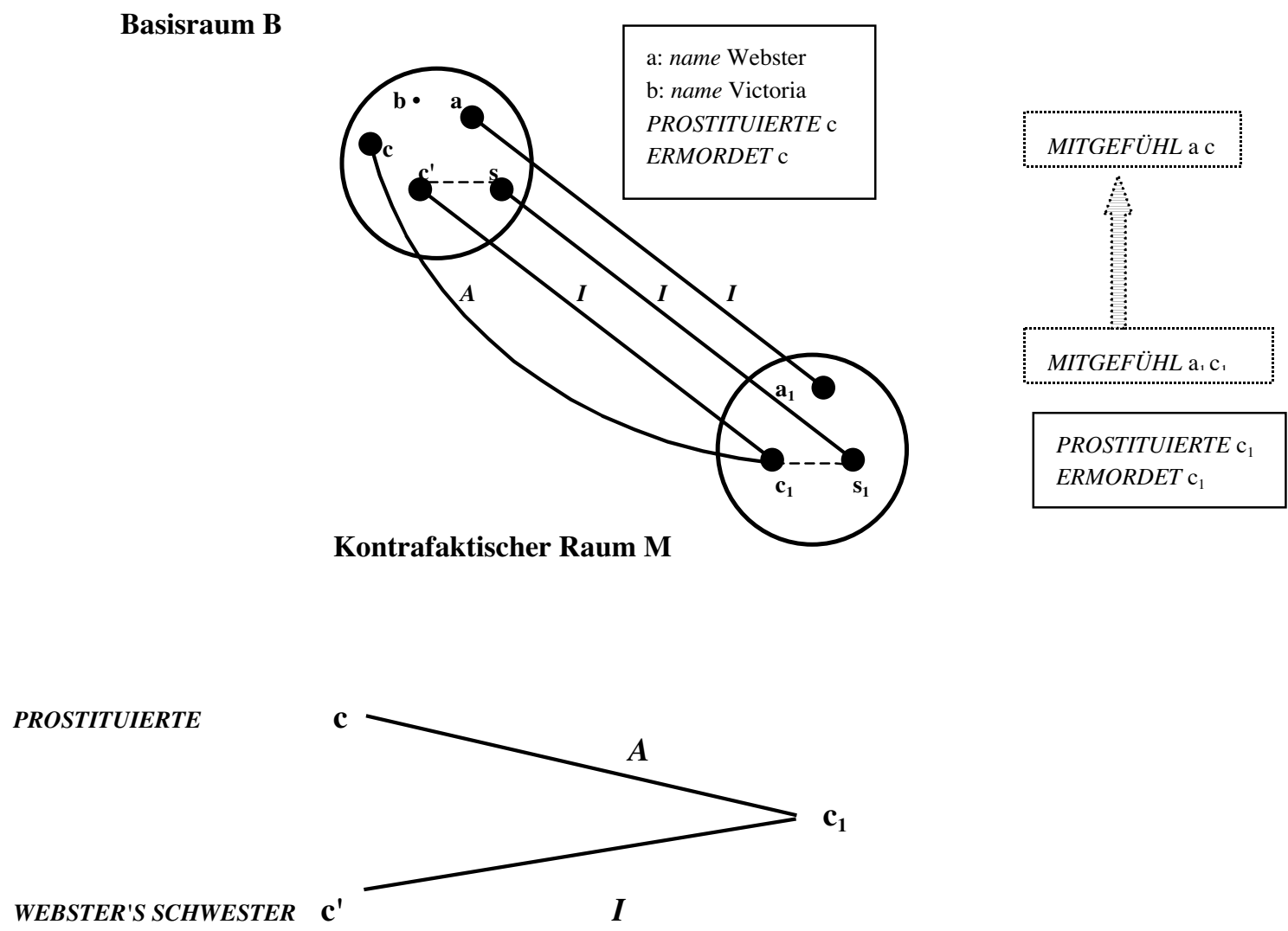

Figur 3

Betrachten wir nun, wie sich die konstruierten mentalen Räume auf die vorbereitenden Bedingungen der durch den ersten Redebeitrag vollzogenen Vorwurfshandlung auswirken.

Wie oben bereits erwähnt, determiniert $\Sigma_{l}$ im konkreten Äußerungskontext i von (2), dass Victoria die Proposition $\mathrm{P}_{1}=\{$ Victoria hat Gründe, Evidenzen, Belege o. Ä. dafür, dass die von ihr thematisierte Proposition \{what if it were your sister?\} sich auf ein bestimmtes Ereignis bezieht, das (tatsächlich) stattgefunden hat und von dem entweder sie oder Webster betroffen bzw. für dessen Zustandekommen entweder der eine oder der andere verantwortlich ist\} als wahr präsupponieren muss. Das präsupponierte Ereignis besteht darin, dass Webster eine bestimmte, von Victoria als erwartbar betrachtete Handlung nicht vollzogen hat: Er hat nämlich beim Anblick der ermordeten Prostituierte kein Mitgefühl gezeigt. In den konstruierten mentalen Räumen versucht Victoria, die Erwartbarkeit der unterlassenen Handlung zu begründen. ${ }^{10}$

Der Sachverhalt der Ermordung wird dabei so dargestellt, dass es prinzipiell nicht ausgeschlossen ist, dass an Stelle der Prostituierten eine beliebig andere Person hätte ermordet werden können. Hierbei könnte es sich folglich auch um die Schwester von Webster handeln (Verbindung $\mathrm{c}-\mathrm{c}_{1}$ ). Bemerkenswert ist allerdings, dass Victoria die Prostituierte nicht einfach durch die wirkliche (bzw. als wirklich präsupponierte) Schwester von Webster ersetzt. Beide Frauen bleiben im Basisraum unabhängig voneinander repräsentiert (cf. c und c'). Sie

\footnotetext{
10 Erwartbarkeit ist naturgemäß die Bedingung der Möglichkeit für die Feststellung einer unterlassenen
} Handlung. 
behauptet also nicht, dass Webster eine Schwester hat, die auch Prostituierte ist und die als

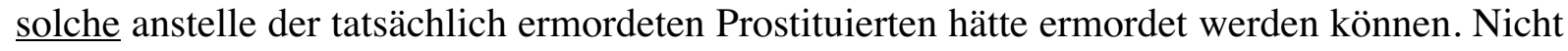
etwa $c^{\prime}$, sondern $c_{1}$ ersetzt $c$. Und $c_{1}$ entsteht teilweise aus Eigenschaften von $c$ und teilweise aus Eigenschaften aus c'. D. h.: Das Element $\mathrm{c}_{1}$ kann nach dem frame-blending effect nicht alle Eigenschaften von $\mathrm{c}$ und auch nicht alle Eigenschaften von c' erhalten.

Zwei Tatsachen sind dabei besonders hervorzuheben. Zunächst ist festzustellen, dass das Element $\mathrm{c}_{1}$ an nur einem der beiden frames teilhat, die mit dem Element $\mathrm{c}$ verbunden sind. ${ }^{11}$ Das für die Repräsentation des fraglichen Sachverhalts im Vordergrund stehende frame ist das frame ERMORDERT. Es ist daher nahe liegend, davon auszugehen, dass dieses frame - und nicht etwa das frame PROSTITUIERTE - von $\mathrm{c}_{1}$ erhalten wird. Dieses frame wird dann allerdings nicht in unveränderter Form von $\mathrm{c}$ an $\mathrm{c}_{1}$ übertragen. Denn beide Elemente instanzieren das frame auf unterschiedliche Art und Weise: Während das Element c für eine Person steht, die tatsächlich ermordet wurde, repräsentiert $c_{1}$ eine Person, die lediglich hätte ermordet werden können. Die erste Form der Instanzierung kann außerdem für $\mathrm{c}_{1}$ schon deshalb nicht infrage kommen, weil sie in Widerspruch zu den Eigenschaften von c' stünde. Das Element c' repräsentiert nicht eine ermordete, sondern eine lebende Person. Das Element $\mathrm{c}_{1}$ zeichnet sich also durch die Rolle "Schwester von Webster" und durch die soeben skizzierte, modifizierte Version des frames ERMORDET aus.

Die Konstruktionsleistung des ersten Redebeitrags zusammenfassend ist festzuhalten, dass Victoria eine analoge Situation bildet, die sich von der realen Situation grundsätzlich nur darin unterscheidet, dass das Element c (ermordete Prostituierte) durch das Element $c_{1}$ charakterisiert durch die soeben genannten Eigenschaften - ersetzt wird. Sie legt damit den Schluss nahe, dass das, was der Prostituierten zugestoßen ist, genauso gut Websters Schwester (als "normale" Frau) hätte zustoßen können. Durch diese Substitution bewirkt sie wie oben bereits erwähnt - die Aktivierung von frames bzw. von kognitiven Modellen des Typs 'man muss Mitgefühl zeigen, wenn die eigene Schwester ermodert wird'. Einmal aktiviert, generieren diese frames und kognitiven Modelle die (von Victoria intendierte) Inferenz: 'wenn einer Frau etwas zustößt (Ermordung), was genauso gut deiner eigenen Schwester (oder dir selber) hätte zustoßen können, ist es (moralisch) erwartbar, dass du Mitgefühl zeigst'.

Die von Victoria im Vollzug ihrer Vorwurfshandlung im ersten Redebeitrag konstruierten mentalen Räume stellen $\Sigma_{1}, \Sigma_{2}$ und $\Sigma_{3}$ als erfüllt dar: Webster hätte beim Anblick der ermordeten Prostituierten Mitgefühl zeigen müssen aber er tat das nicht $\left(\Sigma_{l}\right)$; Victoria ist ('zu Recht') vom Ausbleiben dieser als allgemein erwartbar betrachteten Handlung betroffen $\left(\Sigma_{2}\right)$; und für das Ausbleiben dieser Handlung ist Webster verantwortlich, denn es hätte sich ja genauso gut um seine eigene Schwester handeln können $\left(\Sigma_{3}\right)$. Auf der Grundlage der

\footnotetext{
11 Hier ist die Analyse von Fauconnier m. E. etwas ungenau (cf. oben Figur 3). Denn das frame-blending effect setzt voraus, dass die frames eines Ursprungselements nicht unverändert an das Zielelement übertragen werden können. Die Elemente $\mathrm{c}$ und $\mathrm{c}_{1}$ könnten dementsprechend nicht über die gleichen frames (PROSTITUIERTE, ERMORDET) in unveränderter Form verfügen.
} 
konstruierten mentalen Räume ist der Sprechakt des ersten Redebeitrags - jedenfalls was seine vorbereitenden Bedingungen betrifft - defektfrei vollzogen.

Betrachten wir nun, inwiefern Webster durch seine Nachfolgeäußerung (I don't have a sister, but if I did, she wouldn't be a hooker) die konstruierten mentalen Räume modifiziert und wie sich diese eventuelle Modifikation auf $\Sigma_{1}, \Sigma_{2}$ und $\Sigma_{3}$ auswirkt.

Webster reagiert auf den Redebeitrag von Victoria zunächst mit der Gegenbehauptung "I don't have a sister", die sich explizit auf die Prässuposition bezieht, die durch die definite Struktur "your sister" generiert wurde. ${ }^{12}$ Diese Gegenbehauptung bewirkt die Entfernung des Elements c' aus dem Basisraum, so dass die Rolle s (= "Schwester von Webster") in diesem Raum von keinem Element mehr erfüllt wird. Die Übertragung von Eigenschaften von $c^{\prime}$ an $c_{1}$ wird blockiert und das frame 'man muss Mitgefühl zeigen, wenn die eigene Schwester ermodert wird' folglich auch deaktiviert. Und als letzte Konsequenz kann die (von Victoria intendierte) Inferenz ('wenn einer Frau etwas zustößt (Ermordung), was genauso gut deiner eigenen Schwester (oder dir selber) hätte zustoßen können, ist es (moralisch) erwartbar, dass du Mitgefühl zeigst') nicht mehr erzeugt werden.

Da das Element $\mathrm{c}_{1}$ im kontrafaktischen Raum allerdings noch mit der Rolle $\mathrm{s}_{1}$ verbunden ist, könnte die Inferenz erzeugt werden, dass Webster Mitgefühl zu zeigen hat, nicht, weil seine reale Schwester, sondern weil, 'irgendeine' hypothetische Schwester von ihm an der Stelle der Prostituierten hätte ermordet werden können. Auch diese eventuelle Interpretationsmöglichkeit wird von Webster durch den zweiten Teil seines Beitrags (...but if I did, she wouldn't be a hooker) blockiert. Um das zu erreichen, unternimmt er zweierlei: 1) er modifiziert die Rolle $s_{1}$ (Schwester von Webster) so, dass er ihr die Eigenschaft $\neg$ PROSTITUIERTE zuschreibt und 2) er ersetzt das Element $\mathrm{c}_{1}$ durch ein neues Element $\mathrm{d}_{1}$, das der Eigenschaft $\neg$ PROSTITUIERTE gerecht wird. Aus diesen Modifikationen resultiert, dass das neu eingeführte Element $d_{1}$ - anders als $c_{1}$ - mit dem Element $\mathrm{c}$ des Basisraums nicht verbunden werden kann, da beide Elemente über Eigenschaften verfügen, die sich explizit widersprechen ( $\neg$ PROSTITUIERTE versus PROSTITUIERTE).

Die von Webster so modifizierten mentalen Räume (cf. Figur 4) verhindern jede Möglichkeit der Erzeugung der Inferenz, auf die sich Victoria stützt, um zu zeigen, dass $\Sigma_{1}, \Sigma_{2}$ und $\Sigma_{3}$ erfüllt sind. Denn was der Prostituierten zugestoßen ist, könnten niemals einer (realen oder hypothetischen Schwester) von ihm zustoßen und daher ist es auch nicht erwartbar, dass er beim Anblick der ermordeten Prostituierten Mitleid zeigt $\left(\Sigma_{l}\right)$. Da nicht zutrifft, dass eine allgemein erwartbare Handlung unterlassen wurde, ist es auch nicht gerechtfertigt, dass sich Victoria betroffen zeigt $\left(\Sigma_{2}\right)$. Und schließlich kann Webster für einen Sachverhalt, der einfach nicht besteht, nicht verantwortlich gemacht werden $\left(\Sigma_{3}\right)$. In den von Webster modifizierten mentalen Räumen werden also die vorbereitenden Bedingungen der Vorwurfshandlung nachträglich als unerfüllt dargestellt.

\footnotetext{
12 Eine Gegenbehauptung kann sich nicht nur auf eine (zuvor vom jetztigen Hörer) aufgestellte Behauptung, sondern auch auf eine Prässuposition beziehen. Cf. dazu Huelva Unternbämen (2005).
} 


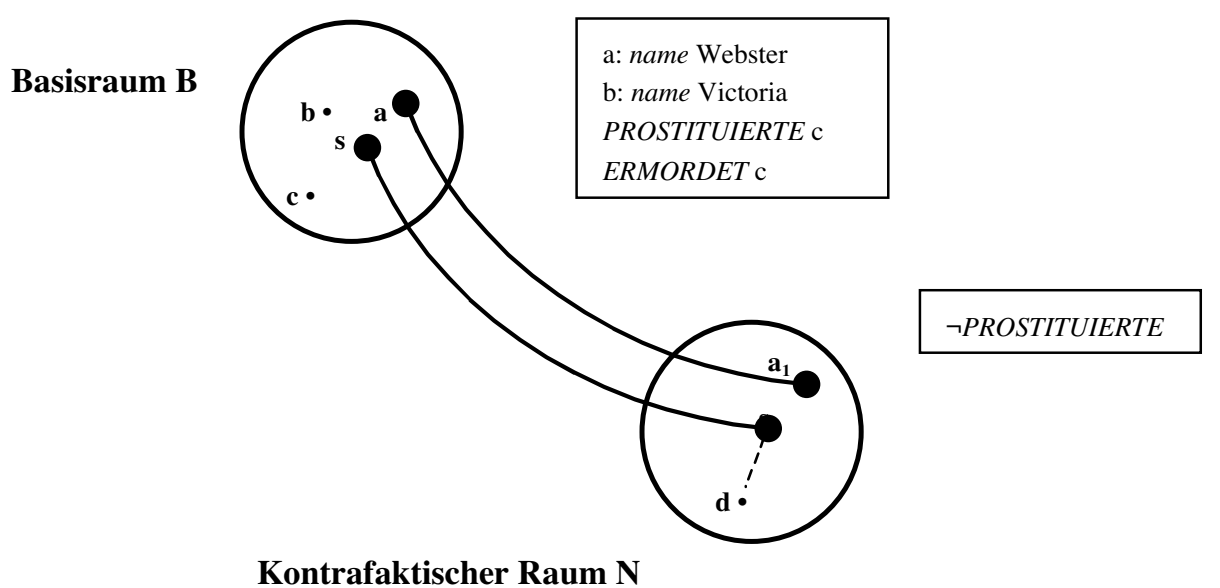

Figur 4

Fasst man die Ergebnisse der Analyse dieses kurzen Beispiels zusammen, so muss man vor allem festhalten, dass die durch beide Gesprächsbeiträge konstruierten mentalen Räume eine unmittelbare Auswirkung auf die vorbereitenden Bedingungen der Vorwurfshandlung von Victoria haben. Während Victoria im Vollzug der Vorwurfshandlung mentale Räume konstruiert, die die vorbereitenden Bedingungen dieser sprachlichen Handlung als erfüllt darstellen, produziert Webster einen Gesprächsbeitrag, der in expliziter Weise zu zeigen versucht, dass diese vorbereitenden Bedingungen keineswegs als erfüllt gelten können. Eine intersubjektive Übereinstimmung hinsichtlich des Erfülltseins der vorbereitenden Bedingungen konnte also nicht herbeigeführt werden.

\section{Theoretisch-methodologische Konsequenzen}

Die erste Konsequenz, die sich aus der vorangehenden Analyse ergibt, ist stricto sensu dialoganalytischer Natur. Das oben analysierte Beispiel besteht aus einer zweigliedrigen Interaktionssequenz, in der der zweite Gesprächsbeitrag explizit die vorbereitenden Bedingungen des Sprechakts thematisiert bzw. problematisiert, der durch den ersten Gesprächsbeitrag ausgeführt wurde. Dieser Sachverhalt exemplifiziert einen Aspekt von Dialogen, der oft in der Dialoganalyse ungenügend berücksichtigt wurde. Nachfolgeäußerungen können sich nicht nur global bzw. holistisch auf den Sprechakt der jeweiligen Voräußerung beziehen. Wie das analysierte Beispiel in aller Deutlichkeit zeigt, sind Interaktanten durchaus in der Lage, unterschiedliche Komponenten von Sprechakten zu identifizieren und gesondert in ihren Äußerungen $\mathrm{zu}$ thematisieren. ${ }^{13} \mathrm{Wie}$ andere Untersuchungen es deutlich zeigen (cf. Huelva Unternbäumen 2005), gibt es sogar bestimmte Sequenztypen, die sich funktional dadurch auszeichnen, dass der Sprecher und/oder der Hörer - meistens durch die Verwendung von bestimmten Konnektoren - eine (semantisch und

\footnotetext{
13 In Huelva Unternbäumen (2005) wird unter anderem ein Fall untersucht, in dem der Sprecher auf die Bedingung des propositionalen Gehalts explizit eingeht.
} 
funktional charakterisierbare) Verbindung zwischen einem Sprechakt und einer einzelnen Komponente eines anderen herstellt.

Die zweite und wichtigere Konsequenz resultiert aus dem Vorschlag, Kontext als eine Abfolge miteinander verbundener mentaler Räume zu betrachten, die von den Interaktanten im Vollzug ihrer Äußerungsakte konstruiert und modifiziert werden. Durch ihre Äußerungen vollziehen Sprecher nicht nur Sprechakte, sondern aktivieren und aktualisieren zugleich Wissensstrukturen in Form von frames und kognitiven Modellen und erzeugen Inferenzen, die zusammen das Hintergrund- bzw. Kontextwissen liefern, das notwendig für den (defektfreien) Vollzug und die Interpretation dieser Akte ist. Äußerungen sind also nicht nur Ausdruckmittel für Illokutionskräfte bzw. für Sprechakte eines bestimmten Typs. Sie sind darüber hinaus auch Instruktionen für die Hervorbringung und Modifikation von mentalen Konstrukten, auf deren Grundlage sie erst vollziehbar und interpretierbar werden.

Am Beispiel der vorbereitenden Bedingungen wurde in dem vorliegenden Beitrag gezeigt, dass die Anwendung der Theorie der mentalen Räumen entscheidend zu einer Operationalisierung des kognitiven Kontextbegriffs beitragen kann. Einerseits ermöglicht sie uns eine explizite und genaue Beschreibung des Inhalts und der Struktur, die der Äußerungskontext als mentales Konstrukt an einer bestimmten Interaktionsstelle besitzt. Und anderseits können wir mit ihrer Anwendung die Dynamik der Prozesse der Hervorbringung und Modifikation dieser mentalen Konstrukte im Laufe des Interaktionsprozesses, sowie die sinnstiftenden Effekte dieser Prozesse detailliert charakterisieren.

\section{Literaturangaben}

Auer, Peter (1986): "Kontextualisierung". Studium Linguistik 19: 22-47.

Auer, Peter (1992): "Introduction: John Gumperz' Approach to Contextualization". In: Auer, Peter/di Luzio, Aldo (eds.): The Contextualization of Language. Amsterdam/Philadelphia: $1-38$.

Davidson, Donald (1985): "Handeln". In: Davidson, Donald: Handlung und Ereignis. Frankfurt am Main: 73-98.

Fauconnier, Gilles (1994): Mental Spaces. Aspects of Meaning Construction in Natural Languages. Cambridge.

Fauconnier, Gilles (1997): Mappings in Thought and Language. Cambridge.

Fauconnier, Gilles/Turner, Mark (1996): "Blending as a Central Process of Grammar". In: Goldberg, Adele (ed.): Conceptual Structure, Discourse, and Language. Stanford: 113130 .

Fauconnier, Gilles/Turner, Mark (1998): "Principles of Conceptual Integration". In: Koenig, Jean-Pierre (ed.): Discourse and Cognition. Stanford: 269-283.

Fauconnier, Gilles/Turner, Mark (2002): The Way We Think: Conceptual Blending and the Mind's Hidden Complexities. New York.

Fillmore, Charles (1982): "Frame Semantics". In: Linguistic Society of Korea (ed.): Linguistics in the Morning Calm. Seoul: 111-138. 
Fillmore, Charles (1985): "Frames and the Semantics of Understanding". Quaderni di Semantica 6,2: 222-253.

Fritz, Gerd/Hundsnurscher, Franz (eds.) (1994): Handbuch der Dialoganalyse. Tübingen.

Gumperz, John (1982): Discourse Strategies. London.

Gumperz, John (1990): "Contextualization and Understanding". In: Duranti, Alessandro/ Goodwin, Charles (eds.): Rethinking Context. Language as an Interactive Phenomenon. Cambridge: 229-252.

Gumperz, John (1992): "Contextualization revisited". In: Auer, Peter/di Luzio, Aldo (eds.): The Contextualization of Language. Amsterdam/Philadelphia: 39-54.

Huelva Unternbäumen, Enrique (2005): "Sprechaktbezogene kausale Satzverknüpfung mit porque im gesprochenen Spanisch". Zeitschrift für romanische Philologie 121. (im Druck).

Hundsnurscher, Franz (1993): "Streitspezifische Sprechakte: Vorwerfen, Insistieren, Beschimpfen". Protosoziologie 4: 140-150.

Kindt, Walther (2002): "Konzeptuelle Grundlagen einer Theorie der Verständigungsprobleme". In: Fiehler, Reinhard (ed.): Verständigungsprobleme und gestörte Kommunikation. Radolfzell: 17-43.

Lakoff, George (1987): Women, Fire, and Dangerous Things. What Categories Reveal about the Mind. Chicago.

Rolf, Eckard (1997): Illokutionäre Kräfte. Grundbegriffe der Illokutionslogik. Opladen.

Searle, John (1983) Sprechakte. Ein sprachphilosophischer Essay. Frankfurt am Main.

Searle, John/Vanderveken, Daniel (1985): Foundations of Illocutionary Logic. Cambridge.

Schnädelbach, Herbert (1977): Reflexion und Diskurs. Fragen einer Logik der Philosophie. Frankfurt am Main.

Vanderveken, Daniel (1988): Les Actes de Discours. Essai de Philosophie du Langage et de l' Esprit sur la Signification des Énonciations. Liege/Bruxelles.

Vanderveken, Daniel (1990): Meaning and Speech Acts. Vol. I: Principles of Language Use. Cambridge. 